\title{
IMAGING OF PULMONARY EDEMA WITH ELECTRICAL IMPEDANCE TOMOGRAPHY
}

\author{
Andy ADLER ${ }^{\dagger}$, Yves BERTHIAUME ${ }^{\ddagger}$, Robert GUARDO ${ }^{\dagger}$, Robert AMYOT* \\ †Institut de Génie Biomédical, Ecole Polytechnique et Université de Montréal, Québec, Canada \\ ¥Centre de Recherche, Hôpital Hôtel Dieu de Montréal, Montréal, Québec, Canada
}

Abstract - Electrical Impedance Tomography (EIT) uses electrical measurements at electrodes placed on the thorax to image changes in the conductivity distribution within the thorax. This technique should be of interest in studying pulmonary edema as the clinical phenomena of interest induce large conductivity changes. We have conducted a series of experiments on dogs to quantify the capacity of EIT to measure lung tidal volume and fluid content. Results indicate good correlation of the quantities measured by EIT with standard reference techniques. Certain difficulties with the long term stability of electrodes and measurement electronics were noted as design criteria for future EIT systems.

\section{Introduction}

Electrical Impedance Tomography (EIT) is an imaging technique which calculates the electrical conductivity distribution within a medium from electrical measurements made from a series of electrodes on the medium surface. We use dynamic EIT (i.e. the measurement of changes in the conductivity distribution, rather than the conductivity itself) because it is better able to compensate for measurement errors, and is more sensitive to small conductivity changes. Images are reconstructed of the change in logarithm conductivity between two sets of measurements taken at times $t_{1}$ and $t_{2}$ using a regularised linear reconstruction algorithm [1]. Thus, if the average image pixel value in the region of the left lung was $x$, the ratio between the lung conductivities at $t_{1}$ and $t_{2}$ will be

$$
\frac{\sigma_{t_{1}}}{\sigma_{t_{2}}}=e^{\left(\log \sigma_{t_{2}}-\log \sigma_{t_{1}}\right)}=e^{x}
$$

\section{Clinical Problem}

The study of the modulation of the recovery phase of pulmonary edema $(\mathrm{PE})$ in humans is difficult since there is no non-invasive technique that would allow a continuous monitoring of the level of edema. In the past, thoracic impedance has been proposed as a tool for monitoring pulmonary edema in vivo; however, because of large variations in the normal values due to the effect of lung volume on the measurement, it was not considered a reliable technique. EIT has been proposed as a technique to monitor $\mathrm{PE}$ while rejecting contributions from other physiological processes. Using the cross sectional image of the change in conductivity in the thorax, it should possible to measure conductivity changes only in the region of interest. EIT is also non-invasive and minimally cumbersome, both of which are important considerations for clinical monitoring equipment.

\section{Experimental Procedure.}

Our EIT data acquisition system uses 16 electrodes for both current injection and voltage measurement, and is able to read the induced voltage at all electrodes for one injection pattern in $40 \mathrm{~ms}$. A current injection of $1 \mathrm{~mA}$ at $13 \mathrm{kHz}$ was used (this is approximately $10 \%$ of the threshold for cutaneous perception in humans.) In order to reject any conductivity changes due to cardiac activity, all data acquisition was gated at $100 \mathrm{~ms}$ after the QRS peak. The electrodes were evenly spaced around the thorax of the animal, approximately $10 \mathrm{~cm}$ above the base of the lungs.

Experiments were conducted on six large dogs. Three diffrerent protocols were used on each animal to measure conductivity change due to lung ventilation, lung fluid instillation, and fluid reabsorbtion in the lungs over time. Experiments were begun one hour after anaesthetisation and hemodynamic preparation of the animal. All EIT data measurements were repeated three times to calibrate for experimental noise.

Lung ventilation was measured by imaging the difference between EIT measurements at end expiration and end inspiration. Measurements were taken at ventilator tidal volumes between $100 \mathrm{~mL}$ and $1000 \mathrm{~mL}$ in $100 \mathrm{~mL}$ increments. All lung ventilation data was aquired before fluid instilation took place.

In order to measure lung fluid content all EIT data was aquired at end expiration at a constant level of lung ventilation, and images were reconstructed relative to a reference taken prior to fluid instillation. Saline solution with added protien (5\% Bovine Albumin Solution) and a coloring marker (Evans Blue) was instilled into a lobe of the right lung through a catheter positioned using a bronchoscope. Measurements were taken after succesively instilling 10, 25, 50,75 and $100 \mathrm{~mL}$ of fluid, and subsequently measurements were taken every $30 \mathrm{~min}$. for the next four hours, to monitor fluid reabsorbtion.

After the last data acquisition, the lungs were extracted and the residual fluid content difference was measured using a gravimetric lung water technique, where the normal lung served as a control to calculate the residual amount of water in the instilled lung. This technique has a coefficient of variation of $10 \%$ [2], and is considered to be a standard for lung water measurement.

\section{Results and Discussion}

Figure 1A shows a typical image of the change in conductivity distribution between end expiration and end inspiration with a tidal volume of $500 \mathrm{~mL}$, while figure $1 \mathrm{~B}$ 
shows the conductivity change due to instilation of $100 \mathrm{~mL}$ saline in the right lung. In order to use the same gray scale mapping for both images, we do not show the fact that the change due to ventilation is non-conductive, while that for instillation is conductive. The EIT images, while of low resolution, show the positions of the lungs, and clearly distinguish the lung which underwent instillation.
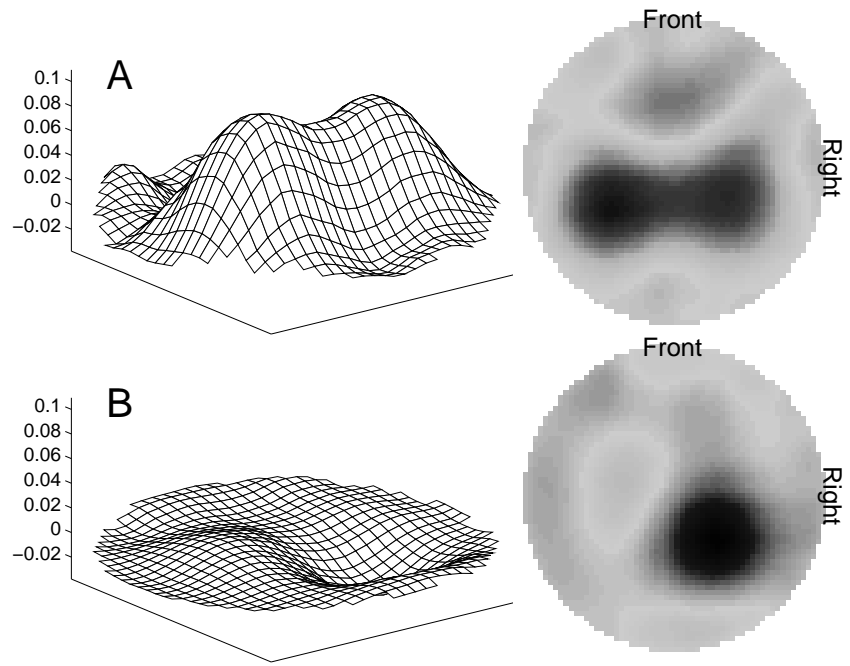

Figure 1: Images of the conductivity change in the thorax

A: Image due to $500 \mathrm{~mL}$ tidal volume

B: Image due to $100 \mathrm{~mL}$ fluid instillation

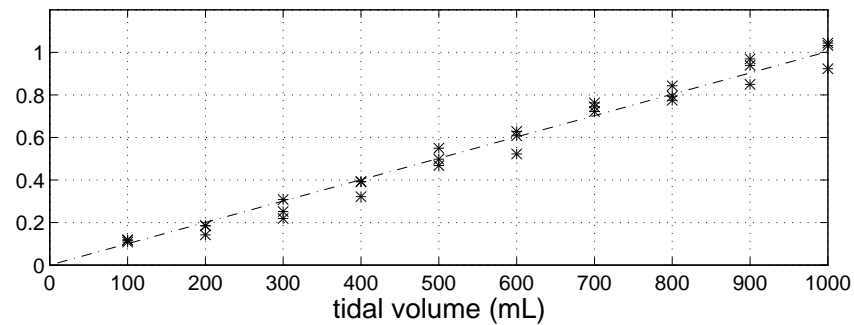

Figure 2: Conductivity change in the thorax from end expiration to end inspiration vs. tidal volume

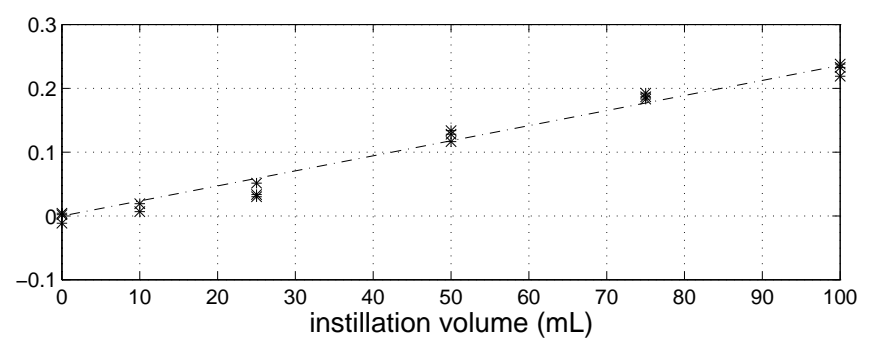

Figure 3: Conductivity change in the thorax vs. instilled fluid quantity

In order to quantify the magnitude of the physiological process, we calculate a parameter set to the sum of all image pixels. Figures 2 and 3 show typical graphs of the magnitude of this parameter versus the tidal volume and instillation fluid volume respectively. Both graphs are normalised with respect to the $100 \mathrm{ml}$ instillation value. The response of the EIT system is linear with respect to these phenomena over the entire measured range, and is sufficiently accurate to reliably detect a $10 \mathrm{~mL}$ change in fluid volume.

EIT image changes due to the resolution of the pulmonary edema were calculated as the difference of the sum of image pixels between the right and left sides of the animal. The change in this parameter over the monitoring period was taken to indicate the percent fluid reabsorbtion in the lung. This corresponds to the calculation by the lung water technique which uses the normal lung as a control for calculating the fluid content of the edematous lung.

The following table presents the results for each of these processes for each animal:

\begin{tabular}{|c|c|c|c|c|}
\hline Dog & Ventilation & Instillation & \multicolumn{2}{|c|}{ Residual Lung Water } \\
& $(r$ coef. $)$ & $(r$ coef. $)$ & EIT & Gravimetric \\
\hline \hline 1 & 0.975 & 0.997 & 0.960 & 0.660 \\
\hline 2 & 0.995 & 0.999 & 1.067 & 0.637 \\
\hline 3 & 0.998 & 0.983 & 0.776 & 0.813 \\
\hline 4 & 0.998 & 0.995 & 0.845 & 0.785 \\
\hline 5 & 0.998 & 0.984 & 0.851 & 0.689 \\
\hline 6 & 0.994 & 0.980 & 0.987 & 0.913 \\
\hline
\end{tabular}

The values indicated for the ventilaton and the instillation is the Pearson $r$ correlation coefficient, where a value of 1 indicates complete linear correlation. The residual water is the fraction of fluid remaining after 4 hours; the value is shown as calculated from the EIT images and from the gravimetric technique. The results for the ventilation and the instillation show very high correlation to the measured physiological phenonema. The variability in measurements is less than the change due to $10 \mathrm{~mL}$ instillation, indicating that EIT is sufficiently sensitive to measure these phenomena.

The calculated reabsorbtion, however, is significantly less accurate; only half of the results are within $10 \%$ of the values obtained by the gravimetric technique. This performance is, at least in part, due to the change in contact quality of the electrodes, and the drift of the measurement electronics with time. In future designs of EIT tomographic systems it is important that the long term stability of the electronics be carefully controlled.

These results indicate that EIT shows promise as an imaging technique for pulmonary physiology. More work, however, is required to improve the long term stability of measurements in order for this technique to be applicable in a clinical monitoring situation.

\section{References}

[1] Adler, R. Guardo, "Electrical Impedance Tomography: Regularised Imaging and Contrast Detection", Submitted to IEEE Trans. Medical Imaging.

[2] Y. Berthiaume, N.C. Staub, M.A. Mathay, "BetaAdrenegic Agonists Increase Lung Liquid Clearance in Anesthetized Sheep", J. Clinical Investigation, pp. 335343, Vol 79, Feb. 1987. 